Article

\title{
Neutrino Signals in Electron-Capture Storage-Ring Experiments
}

\section{Avraham Gal}

Racah Institute of Physics, The Hebrew University, Jerusalem 91904, Israel; avragal@savion.huji.ac.il

Academic Editors: Stefan Frauendorf and Herbert Weigel

Received: 27 December 2015; Accepted: 26 May 2016; Published: 15 June 2016

Abstract: Neutrino signals in electron-capture decays of hydrogen-like parent ions $P$ in storage-ring experiments at GSI are reconsidered, with special emphasis placed on the storage-ring quasi-circular motion of the daughter ions $D$ in two-body decays $P \rightarrow D+v_{e}$. It is argued that, to the extent that daughter ions are detected, these detection rates might exhibit modulations with periods of order seconds, similar to those reported in the GSI storage-ring experiments for two-body decay rates. New dedicated experiments in storage rings, or using traps, could explore these modulations.

Keywords: neutrino interactions, mass and mixing; electron capture

\section{Introduction}

Electron capture (EC) rates for two-body decays $P \rightarrow D+v_{e}$ of hydrogen-like parent ions $P$ coasting in the experimental storage ring of the Heavy-Ion Research Laboratory (GSI) at Darmsrtadt, Germany, exhibit time modulation with laboratory period $\tau_{\mathrm{GSI}} \approx 7 \mathrm{~s}[1,2]$ as shown for ${ }^{142} \mathrm{Pm}$ in Figure 1.

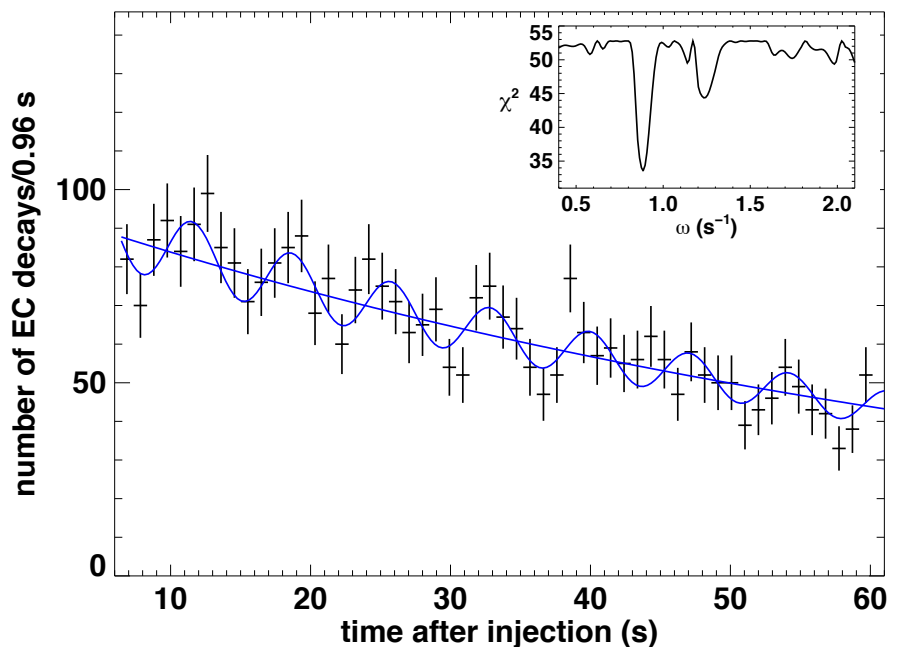

Figure 1. Electron capture (EC) decay rate of $\mathrm{H}-$ like ${ }^{142} \mathrm{Pm}^{60+}$ ions circulating in the GSI experimental storage ring as recorded by a $245 \mathrm{MHz}$ resonator. A fit with angular frequency $\omega=0.884(14) \mathrm{s}^{-1}$, see Equation (1), is also shown, with $\chi^{2}$ values $v s$. $\omega$ given in the inset. Courtesy of Fritz Bosch (figure adapted from [2]).

In Figure 1, the number of EC decays per time interval $d t$ is given, in obvious notation, by

$$
\frac{d N_{\mathrm{EC}}(t)}{d t}=N(0) \exp (-\lambda t) \lambda_{\mathrm{EC}}\left(1+A \cos \left(\omega_{\mathrm{EC}} t+\phi\right)\right)
$$


where $t=0$ marks the time at which the parent ions $P$ were injected to the storage ring. The modulation angular frequency $\omega_{\mathrm{EC}}$ corresponds to an extremely minute energy splitting given by $\hbar \omega_{\mathrm{EC}} \approx 0.83 \times 10^{-15} \mathrm{eV}$ in the parent-ion rest frame. The amplitude $A$ of the reported modulation is $A=0.107 \pm 0.024$ [2], half of that found in the previous experiment [1]. The decay of parent ions in the GSI experiments is signaled, to a variable degree, by the correlated observation of daughter ions which are also confined to a storage-ring motion, as discussed below. In contrast to these storage-ring results, non storage-ring experiments elsewhere found no trace of EC decay-rate modulations $[3,4]$. A new storage-ring experiment, aimed at confirming the reported modulation, has been run recently at GSI [5].

Several works, beginning with the first GSI report [1], noticed that the mass-eigenstate neutrino energies $E_{v_{j}}\left(p_{j}\right)$ in the $P \rightarrow D+v_{j}$ EC decay differ in the parent-ion rest frame $\mathrm{P}$ by

$$
\left|E_{v_{2}}^{(P)}\left(p_{2}\right)-E_{v_{1}}^{(P)}\left(p_{1}\right)\right|=\frac{\Delta m_{v}^{2}}{2 M_{P}}=0.29 \times 10^{-15} \mathrm{eV} \approx \frac{1}{3} \hbar \omega_{\mathrm{EC}},
$$

suggesting perhaps that the reported modulation arises from interference between EC amplitudes $A_{v_{j}}\left(P \rightarrow D+v_{j} ; t\right)$ that encode quantum entanglement of the daughter ion with the mass-eigenstate neutrinos $v_{j}(j=1,2)$ to which the emitted (but undetected) neutrino $v_{e}$ is dominantly coupled. In Equation (2), $\Delta m_{v}^{2} \equiv\left|m_{2}^{2}-m_{1}^{2}\right|=(7.6 \pm 0.2) \times 10^{-5} \mathrm{eV}^{2}$ is the squared-mass difference of these two mass-eigenstate neutrinos [6] and $M_{P} \approx 132 \mathrm{GeV}$ is the rest mass of ${ }^{142} \mathrm{Pm}$. We note that the natural scale for neutrino-oscillation frequencies $\omega_{v}$ is $\hbar \omega_{v}=\Delta m_{v}^{2} / 2 E_{v}$ which, for $E_{v} \sim 4 \mathrm{MeV}$, is about four orders of magnitude larger than the reported $\hbar \omega_{\mathrm{EC}}$. Ivanov and Kienle in particular [7] attempted to derive rigorously $\hbar \omega_{\mathrm{EC}}$ as the underlying energy splitting in two-body EC decays from such interference. However, since mass-eigenstate neutrinos $v_{j}$ are distinct particles, and neutrinos remain undetected in the GSI storage-ring experiments, the general rules of quantum mechanics require to sum up the amplitudes $A_{v_{j}}$ incoherently, $\left|A_{v_{1}}\right|^{2}+\left|A_{v_{2}}\right|^{2}$, with no amplitude interference terms arising in the calculated EC decay rate. This point was forcefully made in Refs. [8-15]. A similar argument for the impossibility to have amplitude interference, and hence time modulations in daughter-ion appearance rates, is briefly reviewed in Section 2.1.

The present work is focused on studying the time evolution of daughter ions $D$ in the GSI storage-ring spatially confined quasi-circular motion. Here, the storage-ring magnetic field disentangles momentarily the daughter ion from the neutrino with which it was produced in the EC decay. At subsequent times the daughter ion and the neutrino are no longer entangled together by the requirement that their total linear momentum vanishes in the parent rest frame $P$. The quasi-circular motion of $D$ is not correlated with the outgoing neutrino rectilinear path, and it is legitimate to treat it independently of the emitted neutrino so long as the remaining constraints imposed by the EC decay kinematics are respected. Furthermore, we note that the GSI technique of recording parent decay events requires the unambiguous identification of the daughter ions over times of order $0.5 \mathrm{~s}$, during which they coast in the storage ring for a total of $\sim 10^{6}$ revolutions while their momentum spread is reduced drastically by the applied electron cooling, as shown in Figure 2 for two daughter-ion frequency trajectories. A key question is whether or not the coherence prevailing at the time of the daughter-ion production could be sustained during the short period before the applied electron cooling might have completely wiped it out. Some comments on this issue, focusing on the role of electron cooling in the GSI storage-ring EC decay experiments [16], are made in Section 3.2.

Provided the answer to the question posed above is positive, meaning that some coherence is sustained, it is shown in Sections 2.2 and 2.3 that the time evolution of daughter ions in their storage-ring quasi-circular confined motion gives rise to modulations with angular frequency close to that given in Equation (2) and thereby close to that reported by the GSI experiments. Such modulations do not arise in rectilinear two-body decays [17]. This aspect of the GSI storage-ring experiments is considered here for the first time. Our prediction may be tested by keeping track of the steady motion of daughter ions in the storage ring under stable electron cooling for as long as 
possible. In this way, the weight with which daughter ions affect the decay-rate measurement could be varied and thereby evaluated.

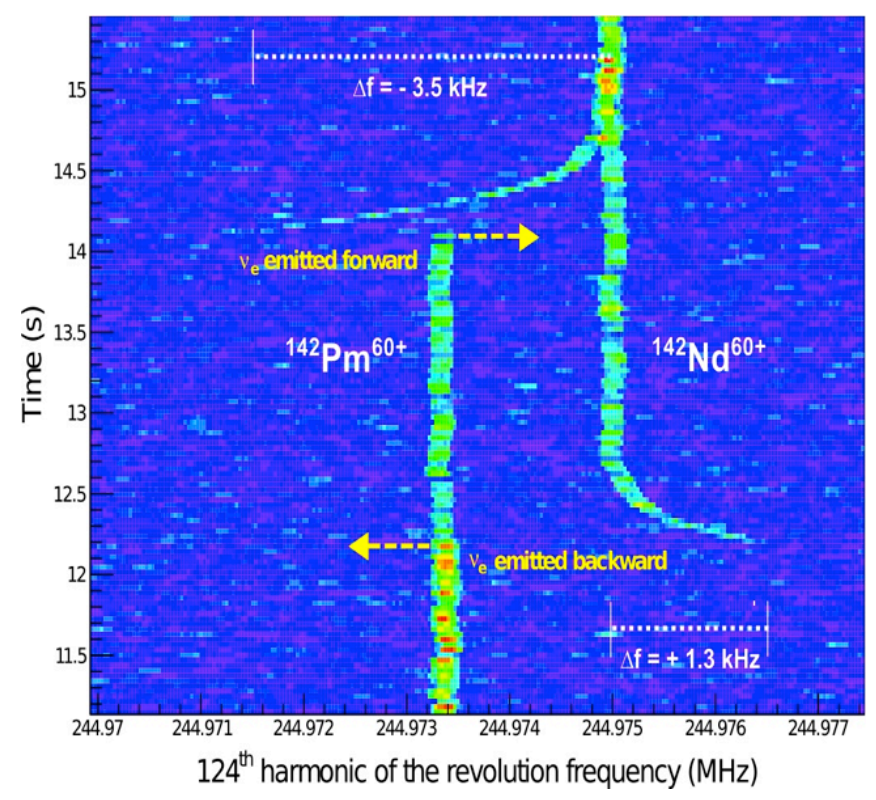

Figure 2. Traces of two cooled ${ }^{142} \mathrm{Pm}^{60+}$ parent ions $v$ s. the time following injection, with time and frequency binning of $32 \mathrm{~ms}$ and $31.25 \mathrm{~Hz}$, respectively. Both parent $\mathrm{H}$-like ${ }^{142} \mathrm{Pm}^{60+}$ ions decay by EC to bare ${ }^{142} \mathrm{Nd}^{60+}$ daughter ions, with arrows indicating the true decay times, as identified by a decrease of the intensity of the trace of the parent ions and the simultaneous onset of the trace of the recoiling daugher ion. The latter starts at a revolution frequency shifted by $\Delta f$ with respect to the frequency after completion of electron cooling. Courtesy of Fritz Bosch (figure adapted from [2]).

\section{Daughter-Ion Oscillations in Two-Body EC}

In the following subsections we confront the standard case in which the daughter-ion motion is not subject to any external electromagnetic (EM) field with that of a daughter-ion quasi-circular storage-ring motion guided by external EM fields.

\subsection{Daughter-Ion Oscillations in Two-Body EC: Non Storage-Ring Motion}

To discuss the disentanglement of a two-body system created at time $t=0$ in EC decay and moving under no external constraints, we briefly recover here the argumentation given in Ref. [9]. The product state $\left|\Psi_{D+v_{e}}(t)\right\rangle$ of $D$ plus electron neutrino $v_{e}$ entangled pair is expanded in the mass-eigenstate neutrino particle basis $v_{j}, j=1,2,3$,

$$
\left|\Psi_{D+v_{e}}(t)\right\rangle=\sum_{j} U_{e j}\left|\psi_{D+v_{j}}(t)\right\rangle
$$

where $U_{e j}$ are elements of the unitary matrix $U$ connecting the electron (flavor) neutrino $v_{e}$ with mass-eigenstate neutrinos $v_{j}$. The state vector $\left|\psi_{D+v_{j}}(t)\right\rangle$ describes the space-time motion of the daughter ion $D$ and mass-eigenstate neutrino $v_{j}$ which are entangled through momentum conservation: $\vec{p}_{D j}+\vec{p}_{v_{j}}=0$ in the parent-ion rest frame P. For simplicity we suppressed the spatial dependence everywhere. The density matrix $\rho_{\Psi}(t)$ associated with $\left|\Psi_{D+v_{e}}(t)\right\rangle$ is given by

$$
\rho_{\Psi}(t)=\left|\Psi_{D+v_{e}}(t)\right\rangle\left\langle\Psi_{D+v_{e}}(t)\right| .
$$


The observation of the daughter ion $D$ at a given time $t>0$ disentangles the two-body system. This requires to project out the undetected neutrino. To this end one defines an appropriate projection operator

$$
Q_{v}=\sum_{j} \int d^{3} p_{v}\left|\psi_{D+v_{j}}, \vec{p}_{v}\right\rangle\left\langle\psi_{D+v_{j}}, \vec{p}_{v}\right|
$$

where the sum and integral run over a complete set of mass-eigenstate neutrinos $v_{j}$ with momenta $\vec{p}_{v}$. The summation on $v_{j}$ could in principle be replaced by summing on flavor neutrinos $v_{\alpha}$, demonstrating that $Q_{v}$ is flavor blind. Taking the trace of $Q_{v} \rho_{\Psi}(t)$ in the $v_{j}$ basis and also integrating over the neutrino phase space gives

$$
\operatorname{Tr}_{v}\left(Q_{\nu} \rho_{\Psi}(t)\right)=\sum_{j}\left|U_{e j}\right|^{2}=1,
$$

where the orthogonality of mass-eigenstate neutrinos, $\left\langle v_{j} \mid v_{j^{\prime}}\right\rangle=\delta_{j j^{\prime}}$, was instrumental to eliminate interference terms that could have led to daughter ion $D$ oscillations.

\subsection{Daughter-Ion Oscillations in Two-Body EC: Storage-Ring Motion I}

As argued in the Introduction, the external magnetic field that confines $D$ in its circular motion disentangles $D$, for each of its equal-mass energy-momentum components $D_{j}$, from the propagating mass-eigenstate neutrinos $v_{j}$. This occurs momentarily upon EC decay, following which the space-time motion of the daughter ion $D$ and mass-eigenstate neutrinos $v_{j}$ is no longer entangled through momentum conservation: $\vec{p}_{D j}+\vec{p}_{v_{j}}=0$. Detection of $D$ occurs at a later time. It is justified then to focus on the storage-ring motion of $D$, disregarding the undetected neutrino trajectory except through the dependence of the circular-motion temporal phase $E_{D j} \tau$ (see below) on the $v_{j}$ neutrino label $j$. Here, $\tau=t-t_{d}$ denotes time $t$ with respect to the EC decay time $t_{d}$. To disentangle, we first project the state vector $\left|\Psi_{D+v_{e}}(t)\right\rangle$, Equation (3), on the produced electron neutrino state vector $\left|v_{e}(t)\right\rangle$ in the limit $t \rightarrow t_{d}$, and propagate the $D$ initial state vector $\left|\Psi_{D}\left(t_{d}\right)\right\rangle$ forward in time, thereby obtaining

$$
\left|\Psi_{D}(\vec{x}, t)\right\rangle=\mathcal{N}_{D} \sum_{j}\left|U_{e j}\right|^{2}\left|\psi_{D j}(\vec{x}, t)\right\rangle,
$$

where the spatial $(\vec{x})$ dependence was made explicit and $\mathcal{N}_{D}$ is a space-time independent normalization constant. The state-vector $\left|\psi_{D j}\right\rangle$ wave packet $\psi_{D j}$ is given, in a plane-wave limit, by

$$
\psi_{D j}(\vec{x}, t)=\exp \left[i\left(\vec{p}_{D j} \cdot \vec{\xi}-E_{D j} \tau\right)\right]
$$

per each mass-eigenstate neutrino $v_{j}$ with which $D$ was entangled at the EC decay time $t_{d}$. Here, $\vec{\xi}=\vec{x}-\vec{x}_{d}$ and $\tau=t-t_{d}$. Using a more realistic wave packet rather than a plane wave for $\psi_{D j}$ does not change the nature of conclusions reached below. To obtain the amplitude $A_{D}$ for detecting $D$ at time $t$, subsequent to its production at time $t_{d}$ in the two-body EC decay $P \rightarrow D+v_{e}$, we form the matrix element between the $D$ state vector $\left|\Psi_{D}(\vec{x}, t)\right\rangle$ and the initial state vector $\left|\Psi_{D}\left(\vec{x}_{d}, t_{d}\right)\right\rangle$, normalizing it so that $A_{D}\left(t=t_{d}\right)=1$. The amplitude $A_{D}$ generated in this way is essentially identical with Equation (7):

$$
A_{D}=\sum_{j=1,2,3}\left|U_{e j}\right|^{2} \psi_{D j}(\vec{x}, t)
$$

The space-time wave packets $\psi_{D j}(\vec{x}, t)$ in Equation (9) are weighed by the probabilities $\left|U_{e j}\right|^{2}$ with which each $D_{j}$ was produced at time $t_{d}$ of the EC decay. The use of probabilities, $\sum_{j=1,2,3}\left|U_{e j}\right|^{2}=1$, rather than amplitudes $U_{e j}$ or their complex conjugates $U_{e j}^{*}$, is consistent with the assumption that the daughter ion $D$ was disentangled momentarily by the action of the external magnetic field from the electron neutrino $v_{e}$ with which it had been entangled at the moment of EC decay. Forming the probability $\left|A_{D}\right|^{2}$ gives rise to interference terms $\psi_{D i}^{*} \psi_{D j}, i \neq j$, that generate oscillations, unless 
these interference terms come out independent of space and time as it happens in rectilinear motion; see below.

The appearance of the probabilities $\left|U_{e j}\right|^{2}$ in Equation (9) is reminiscent of a $v_{e} \rightarrow v_{e}$ amplitude for neutrino detection with no flavor change [17]. To check that the corresponding amplitude,

$$
A_{v_{e}}=\sum_{j=1,2,3}\left|U_{e j}\right|^{2} \psi_{v_{j}}(\vec{x}, t),
$$

is also obtainable in the present formalism one projects the state vector $\left|\Psi_{D+v_{e}}(t)\right\rangle$, Equation (3), on the daughter-ion initial state vector $\left|\Psi_{D}\left(t_{d}\right)\right\rangle$, resulting in

$$
\left|\Psi_{v}(\vec{x}, t)\right\rangle=\mathcal{N}_{v} \sum_{j} U_{e j}\left|\psi_{v_{j}}(\vec{x}, t)\right\rangle
$$

Forming the matrix element between the $v$ state vector $\left|\Psi_{v}(\vec{x}, t)\right\rangle$ and the state vector $\left|\Psi_{v_{e}}\left(\vec{x}_{d}, t_{d}\right)\right\rangle$, and normalizing so that $A_{v_{e}}\left(t=t_{d}\right)=1$, one obtains the amplitude $A_{v_{e}}$ in the form Equation (10), as anticipated for it.

\subsection{Daughter-Ion Oscillations in Two-Body EC: Storage-Ring Motion II}

Adopting the idealized plane wave form given by Equation (8) in the parent-ion rest frame P, and assuming rectilinear motion of the stable daughter ion $D$ produced in the two-body decay $P \rightarrow D+v_{e}$, it was shown in Ref. [17] that to order $\mathcal{O}\left(\Delta m_{v}^{2}\right)$ the $j$ dependence of the spatial phase $\vec{p}_{D j} \cdot \vec{\xi}$ cancels the $j$ dependence of the temporal phase $E_{D j} \tau$. This leads to $\psi_{D i}^{*} \psi_{D j}=1$ for all $i$ and $j$. Owing to Lorentz covariance of the space-time phase in Equation (8), $\psi_{D j}(\vec{x}, t)$ is to an excellent approximation independent of $j$ for rectilinear motion in any inertial coordinate frame boosted from the parent-ion rest frame P. Hence, a rectilinearly outgoing daughter-ion $D$ should incur no modulation or oscillation.

The GSI experiment differs in one essential respect from EC experiments done with rectilinear geometry [3,4]. In the GSI experiment the daughter ion traverses a storage-ring quasi-circular trajectory. In this case, because $D$ is confined to the storage ring, it can be shown that interference effects arising from the phases $\vec{p}_{D j} \cdot \vec{\xi}$ are negligible although each of these phases need not be particularly small. Thus, recalling that

$$
p_{D j}^{(P)}-p_{D j^{\prime}}^{(P)}=p_{v_{j^{\prime}}}^{(P)}-p_{v_{j}}^{(P)}=\frac{\Delta m_{j j^{\prime}}^{2}}{2 E_{v}} \quad\left(\Delta m_{j j^{\prime}}^{2} \equiv m_{j}^{2}-m_{j^{\prime}}^{2}\right)
$$

in the parent-ion rest frame $\mathrm{P}$, with the same order of magnitude in the laboratory frame $\mathrm{L}$, and using a spatial size of $\xi \sim 50 \mathrm{~m}$, the difference of two such phases is negligible, $\left|\left(p_{D j}-p_{D j^{\prime}}\right) \xi\right| \ll 1$, and will be disregarded henceforth in considering potential contributions to interference terms. This result follows also upon replacing the plane wave Equation (8) by a wave packet that reflects the storage ring confined motion. Suppressing therefore $\vec{p}_{D j} \cdot \vec{\xi}$ spatial phases, a nonvanishing $j$ dependence emerges from the phase $E_{D j} \tau$. Assuming that no further $j$ dependence arises from the gone-away neutrino, and using Equation (2), interference terms between $\psi_{D j}(x \approx 0, t)$ and $\psi_{D j^{\prime}}(x \approx 0, t)$ for $j \neq j^{\prime}$ are given in the parent-ion rest frame $\mathrm{P}$ by

$$
2 \cos \left(\Delta E_{D j j^{\prime}}^{(P)} \tau_{P}\right)=2 \cos \left(\frac{\Delta m_{j j^{\prime}}^{2}}{2 M_{P}} \tau_{P}\right)
$$

where the rest-frame time $\tau_{P}=0$ corresponds to the EC decay time, and

$$
\Delta E_{D j j^{\prime}}^{(P)} \equiv E_{D j}^{(P)}-E_{D j^{\prime}}^{(P)}=E_{v_{j^{\prime}}}^{(P)}\left(p_{v_{j^{\prime}}}\right)-E_{v_{j}}^{(P)}\left(p_{v_{j}}\right)=\frac{\Delta m_{j^{\prime} j}^{2}}{2 M_{P}},
$$


see also Equation (2). We note that these interference terms are evaluated at a given space-time point $(x \approx 0, t)$, regardless of any space-time path that might have been used to lead to the amplitude $A_{D}$ of Equation (9). The angular frequency suggested by Equation (13) for daughter-ion oscillations comes close indeed to the GSI value $\omega_{\mathrm{EC}}$. A more rigorous derivation of this rest-frame result is provided in Section 3.1, and the transition to the lab frame is detailed in Section 3.2. Unlike transformations of decay rates which require only the well tested $t_{L}=\gamma t_{P}$ relationship between the lab time $t_{L}$ and the proper time $t_{P}$ [18], a more careful consideration of Lorentz transformations involving momentum-energy and space-time is needed here.

\section{Results}

\subsection{Center-of-Mass Oscillation Expressions}

As argued in the Introduction, the external magnetic field that confines $D$ to a circular motion and the application of electron cooling in fact disentangle $D$, for each of its energy-momentum components $D_{j}$, from the propagating mass-eigenstate neutrinos $v_{j}$. This allows us to focus on the storage-ring motion of $D$, disregarding the undetected neutrino trajectory except implicitly through the dependence of the circular-motion phase $E_{D j} \tau$ in Equation (8) on the $v_{j}$ neutrino label $j$. Consider then the amplitude $A_{D}$ Equation (9) for detecting $D$ at time $t$ subsequent to its production at time $t_{d}$ in the two-body EC decay $P \rightarrow D+v_{e}$. Recalling that $\psi_{D j}(\vec{x}, t)$, Equation (8), reduces to $\exp \left(-i E_{D j}^{(P)} \tau\right)$, $A_{D}$ is given in the parent-ion rest frame $\mathrm{P}$ by

$$
A_{D}=\sum_{j=1,2,3}\left|U_{e j}\right|^{2} \exp \left(-i E_{D j}^{(P)} \tau_{P}\right), \quad \tau=t-t_{d} \geq 0,
$$

where $U_{e j}$ are elements of the unitary matrix $U$ connecting the electron (flavor) neutrino $v_{e}$ with mass-eigenstate neutrinos $v_{j}$. Specializing to a $2 \times 2$ orthogonal neutrino mixing matrix with angle $\theta$, the relevant probability $\left|A_{D}\right|^{2}$ is given by

$$
\left|A_{D}\right|^{2}=1-\sin ^{2}(2 \theta) \sin ^{2}\left(\frac{\Delta E_{D 12}^{(P)}}{2} \tau_{P}\right)
$$

which exhibits the same oscillation angular frequency $\Delta E_{D 12}^{(P)}$ discussed in the previous subsection. The structure of Equation (16) coincides, fortuitously though, with the well known neutrino deficit formula for $\left|A_{v_{e}}\right|^{2}$, where $A_{v_{e}}$ is defined by Equation (10), in a $v_{e}$ disappearance experiment. Equation (16), which assumes a given parent-ion decay time $t_{d}$, or equivalently daughter-ion appearance time $t_{d}$, does not yet give the differential rate for EC decay marked by detection of the daughter ion. One needs first to let $t_{d}$ vary between injection time $t_{d}=0\left(\tau_{P}=t_{P}\right)$ to detection time $t_{d}=t\left(\tau_{P}=0\right)$. Hence, we integrate over $\tau_{P}$ between 0 to $t_{P}$ and then differentiate with respect to $t_{P}$ to get the required $D$ detection rate. Note that we have disregarded the exponential intensity depletion of the decay ion at time $t_{d}$ which, in a more systematic derivation, leads to the appearance of the familiar $\lambda \exp \left(-\lambda t_{P}\right)$ prefactor in all of our subsequent $D$ detection rates. This results in precisely the same expression for the daughter-ion detection rate as Equation (16):

$$
R_{D}\left(t_{P}\right)=\left|A_{D}\left(t_{P}\right)\right|^{2}=1-\sin ^{2}(2 \theta) \sin ^{2}\left(\frac{\Delta E_{D 12}^{(P)}}{2} t_{P}\right)
$$

with $\Delta E_{D 12}^{(P)}=\Delta m_{v}^{2} / 2 M_{P}$, see Equation (13) and the text that follows it. Recall that $\Delta E_{D 12}^{(P)}$ was obtained for storage-ring confined motion of $D$. As discussed in the previous subsection, the phase $\Delta E_{D 12}^{(P)} \tau_{P}$ gets canceled in rectilinear motion by a spatial contribution that is suppressed in the GSI storage-ring motion. Therefore, to extrapolate the storage-ring rate Equation (17) to EC rectilinear 
motion, one substitutes formally $\Delta E_{D 12}^{(P)}=0$, obtaining thereby $R_{D}\left(t_{P}\right)_{\text {rectilinear }}=1$, in agreement with standard discussions of recoil oscillations [17]. Equation (17) exhibits modulations with amplitude

$$
A=\frac{\sin ^{2}(2 \theta) / 2}{1-\sin ^{2}(2 \theta) / 2} \approx 0.733 \pm 0.021
$$

relative to a base line of $1-\sin ^{2}(2 \theta) / 2 \approx 0.577 \pm 0.011$, where the value $\sin ^{2}(2 \theta)=0.846 \pm 0.021$ [6] was used. This summarizes our prediction for future experiments focusing on daughter-ion oscillations in confined motion.

\subsection{Laboratory Frame Oscillation Expressions}

In the laboratory frame L, the plane wave Equation (8) assumes the form

$$
\psi_{D j}^{(L)}\left(\vec{x}_{L}, t_{L}\right)=\exp \left[i\left(\vec{p}_{D j}^{(L)} \cdot \vec{\xi}_{L}-E_{D j}^{(L)} \tau_{L}\right)\right]
$$

which upon suppressing the spatial phase $\vec{p}_{D j}^{(L)} \cdot \vec{\xi}_{L}$ gives rise to a lab interference term

$$
2 \cos \left(\Delta E_{D j j}^{(L)} \tau_{L}\right)
$$

with oscillation angular frequency that corresponds to a lab energy splitting $\Delta E_{D j j^{\prime}}^{(L)} \equiv E_{D j}^{(L)}-E_{D j^{\prime}}^{(L)}$. This is evaluated by transforming from the rest frame $\mathrm{P}$ to the lab frame $\mathrm{L}$,

$$
\Delta E_{D j j^{\prime}}^{(L)}=\gamma \Delta E_{D j j^{\prime}}^{(P)}+\gamma \vec{\beta} \cdot \Delta \vec{p}_{D j j^{\prime \prime}}^{(P)}
$$

where $\Delta \vec{p}_{D j j^{\prime}}^{(P)} \equiv \vec{p}_{D j}^{(P)}-\vec{p}_{D j^{\prime}}^{(P)}$ and $\beta=0.71$ with $\gamma \equiv\left(1-\beta^{2}\right)^{-1 / 2}=1.42$ [1,2]. This Lorentz transformation holds everywhere on the storage-ring trajectory of $D$. Recalling from Equations (12) and (14) that $\left|\Delta E_{D j j^{\prime}}^{(P)} / \Delta p_{D j j^{\prime}}^{(P)}\right|=E_{v} / M_{P} \ll 1$, one could argue that the first term on the right-hand side of Equation (21) may be safely neglected with respect to the second one which by Equation (12) is of order $\Delta m_{v}^{2} / 2 E_{v}$, several orders of magnitude larger than $\hbar \omega_{\mathrm{EC}}$. This would render unobservable the oscillations of daughter ions coasting in the GSI storage ring. At this point we recall that the electron cooling applied at the GSI storage ring [16] primarily affects the longitudinal momentum of $D$, parallel to the beam direction $\hat{\beta}$, through small-angle Coulomb scattering, as demonstrated in Figure 2 for the two trajectories depicted for $D$. This is likely to cause partial decoherence by removing the distinction between the separate longitudinal components $p_{\| D j}$. We therefore impose the condition $\vec{\beta} \cdot \Delta \vec{p}_{D j j^{\prime}}^{(P)}=0$, resulting thereby in

$$
\Delta E_{D j j^{\prime}}^{(L)}=\gamma \Delta E_{D j j^{\prime}}^{(P)}=\frac{\gamma \Delta m_{j^{\prime} j}^{2}}{2 M_{P}}
$$

Using a similar reasoning, $\Delta p_{D j j^{\prime}}^{(L)}$ too comes out as small, $\Delta p_{D j j^{\prime}}^{(L)}=\beta \gamma \Delta m_{j j^{\prime}}^{2} / 2 M_{P}$.

Substituting $\Delta E_{D j j^{\prime}}^{(L)}$ from Equation (22) in the interference term Equation (20) leads to the following period of daughter-ion oscillations in the lab frame L:

$$
T_{\mathrm{osc}}^{(L)}=\frac{4 \pi M_{P}}{\gamma \Delta\left(m_{v}^{2}\right)}=10.17 \pm 0.26 \mathrm{~s},
$$

where the uncertainty is due to the uncertainty in the value of $\Delta\left(m_{v}^{2}\right)$. Compared with the reported value of $7.12 \pm 0.11 \mathrm{~s}$ [2], we miss just a factor $(10.17 \pm 0.26) /(7.12 \pm 0.11)=1.43 \pm 0.06$ consistent 
with $\gamma=1.42$. However, this coincidence might well prove fortuitous. Minute departures from the assumed $\vec{\beta} \cdot \Delta \vec{p}_{D j j^{\prime}}^{(P)}=0$ could lead to appreciable modifications of Equation (22) for $\Delta E_{D j j^{\prime}}^{(L)}$.

To demonstrate the sensitivity of the result, Equation (22), to decoherence effects arising from electron cooling, we suppress the kinetic energy contribution to $\Delta E_{D j j^{\prime}}^{(L)}$ from the longitudinal components $p_{\|}^{(L)}$, keeping only the small transverse components $p_{\perp}^{(L)}=p^{(P)} \sin \theta_{P}$ which are less likely to be affected by the dominantly small-angle electron-cooling Coulomb scattering. Thus, retaining only $\Delta p_{\perp D j j^{\prime}}^{(L)}$ contributions, we obtain

$$
\Delta E_{D j j^{\prime}}^{(L)} \approx \frac{p^{(P)} \sin \theta_{P}}{E_{D}^{(L)}} \Delta p_{D j j^{\prime}}^{(P)} \sin \theta_{P}=\frac{\Delta m_{j j^{\prime}}^{2}}{2 \gamma M_{D}} \sin ^{2} \theta_{P} \rightarrow \frac{\Delta m_{j j^{\prime}}^{2}}{3 \gamma M_{D}},
$$

where in the last step we averaged over $\sin ^{2} \theta_{P}$. This $\Delta E_{D j j^{\prime}}^{(L)}$ agrees within a factor of approximately 3 with that of Equation (22). The main message is that the relevant lab energy differences $\Delta E_{D j j^{\prime}}^{(L)}$ remain of the same order as above, $\Delta m_{v}^{2} / 2 M$, and do not vanish.

\section{Conclusions}

In this work, we derived a nonvanishing oscillation frequency that a daughter ion moving in a storage ring should exhibit when disentangled by the action of the guiding external magnetic field from the electron-neutrino produced in the two-body EC decay of a parent ion coasting in the storage ring. This frequency invloves the neutrinos squared-mass difference $\Delta m_{v}^{2}$ divided by $2 M_{P}$, commensurate with the reported GSI EC decay rate modulation frequency $\omega_{\mathrm{EC}} \approx 1 \mathrm{~s}^{-1}$. It is specific to storage-ring motion, vanishing in rectilinear daughter-ion motion as discussed in Ref. [17]. The precise value of the period $T_{\text {osc }}^{(L)}$ given under idealized conditions by Equation (23) depends sensitively on the extent to which the motion of the daughter ion, incorporating its $D_{j}$ components, keeps coherent under given experimental constraints, primarily determined by the electron cooling system performance. In the unrealistic limit of full coherence, upon disregarding electron cooling, the related energy difference from Equation (21) is $\Delta m_{v}^{2} / 2 E_{v}\left(\gg \Delta m_{v}^{2} / 2 M_{P}\right)$ which leads to unobervable oscillations with a period of order $1 \mathrm{~ms}$.

Daughter-ion oscillations in storage-ring circular motion, as discussed above, do not contradict any of the assertions made in Refs. [8-15] that oscillations cannot show up in parent-ion $P$ decay rates or in daughter-ion $D$ appearance rates. In these works disentanglement is triggered by observing the EC decay, at which time the daughter ion $D$ and the undetected electron neutrino $v_{e}$ are entangled with each other, whereas the observation of $D$ in storage-ring circular motion guided by external EM fields occurs when $D$ is no longer entangled with $v_{e}$ from which it was disentangled at the $P \rightarrow D+v_{e}$ decay time. Therefore, none of these assertions hold for a storage-ring motion of the daughter ions.

As long as GSI-like storage-ring EC decay experiments do not distinguish between observables pertaining to decay parent ions and observables associated with circulating daughter ions, daughter-ion oscillations will persist in giving rise to apparent modulation of EC decay rates, with amplitudes that depend on how the decay of parent ions $P$ and the subsequent storage-ring motion of daughter ions $D$ are both handled by the experimental setup and the final analysis of the experiment. The role of electron cooling in possibly preserving some of the coherence in the daughter-ion propagation at the GSI storage ring needs to be studied further, both experimentally and theoretically.

Acknowledgments: I would like to thank Fritz Bosch, Hans Feldmeier, Eli Friedman and Berthold Stech for making critical but useful remarks on several occasions during the preparation of previous versions. Stimulating discussions with Boris Kayser and Murray Peshkin during the recent EMMI Rapid Reaction Task Force workshop on Non-Exponential Two-Body Weak Decays at Jena, Germany, July 2014, are gratefully acknowledged. I also thank Fritz Bosch, Thomas Faestermann, Yuri Litvinov and Thomas Stöhlker, who organized this workshop, for their kind hospitality and support. 
Conflicts of Interest: The author declares no conflicts of interest.

\section{References and Notes}

1. Litvinov, Yu.A.; Bosch, F.; Winckler, N.; Boutin, D.; Essel, H.G.; Faestemann, T.; Geissel, H.; Hess, S.; Kienle, P.; Knöbel, R; et al. Observation of non-exponential orbital electron capture decays of hydrogen-like ${ }^{140} \mathrm{Pr}$ and ${ }^{142}$ Pm ions. Phys. Lett. B 2008, 664, 162-168.

2. Kienle, P.; Bosch, F.; Bühler, P.; Faestemann, T.; Litvinov, Yu.A.; Sanjari, M.S.; Shubina, B.B.; Winckler, N.; Atanasov, D.; Geissel, H.; et al. (Two-Body-Weak-Decays Collaboration). High-resolution measurement of the time-modulated orbital electron capture and of the $\beta^{+}$decay of hydrogen-like ${ }^{142}$ Pm ions. Phys. Lett. B 2013, 726, 638-645.

3. Vetter, P.A.; Clark, R.M.; Dvorak, J.; Freedman, S.J.; Gregorich, K.E.; Jeppesen, H.B.; Mittelberger, D.; Wiedeking, M. Search for oscillation of the electron-capture decay probability of ${ }^{142} \mathrm{Pm}$. Phys. Lett. B 2008, 670, 196-199.

4. Faestermann, T.; Bosch, F.; Hertenberger, R.; Maier, L; Krücken, R.; Rugel, G. Could the GSI decay rate oscillations be observed in a standard electron capture decay experiment? Phys. Lett. B 2009, 672, 227-229.

5. Piotrovski, J.; Chen, X.; Litvinov, Yu.A.; Sanjari, M.S.; Two-Body-Weak-Decays collaboration. Performance of the ESR kicker magnet during E082. GSI Scientific Report 2014, unpublished work. 2015, doi:10.15120/GR-2015-1-FG-GENERAL-11.

6. Abe, K.; Hayato, Y.; Iida, T.; Ikeda, M.; Ishihara, G.; Iyogi, K.; Kameda,J.; Kobayashi, K.; Koshio, Y.; Kozuma, Y.; et al. (Super-Kamiokande Collaboration). Solar neutrino results in Super-Kamiokande-III. Phys. Rev. D 2011, 83, 052010.

7. Ivanov, A.N.; Kienle, P. Time Modulation of the K-Shell Electron Capture Decay Rates of H-like Heavy Ions at GSI Experiments. Phys. Rev. Lett. 2009, 103, 062502.

8. Giunti, C. Rates of processes with coherent production of different particles and the GSI time anomaly. Phys. Lett. B 2008, 665, 92-94.

9. Kienert, H.; Kopp, J.; Lindner, M.; Merle, A. The GSI anomaly. J. Phys. Conf. Ser. 2008, 136, 022049.

10. Cohen, A.G.; Glashow, S.L.; Ligeti, Z. Disentangling neutrino oscillations. Phys. Lett. B 2009, 678, 191-196.

11. Merle, A. Why a splitting in the final state cannot explain the GSI-Oscillations. Phys. Rev. C 2009, 80, 054616.

12. Flambaum, V.V. Comment on "Time Modulation of the K-Shell Electron Capture Decay Rates of H-like Heavy Ions at GSI Experiments". Phys. Rev. Lett. 2010, 104, 159201.

13. Wu, J.; Hutasoit, J.A.; Boyanovsky, D.; Holman, R. Dynamics of disentanglement, density matrix, and coherence in neutrino oscillations. Phys. Rev. D 2010, 82, 013006.

14. Gal, A. Neutrino magnetic moment effects in electron-capture measurements at GSI. Nucl. Phys. A 2010, 842, 102-112.

15. Peshkin, M. Oscillating decay rate in electron capture and the neutrino mass difference. Phys. Rev. C 2015, 91, 042501.

16. Steck, M.; Beller, P.; Beckert, K.; Franzke, B.; Nolden, F. Electron cooling experiments at the ESR. Nucl. Instrum. Methods A 2004, 532, 357-365.

17. Kayser, B. Neutrino Oscillation Physics. In Proceedings of the International School of Physics "Enrico Fermi"; Bellini, G., Ludhova, L., Eds., IOS Press: Amsterdam, The Netherlands, 2012; pp. 1-14.

18. For a recent discussion of decay-rate frame dependence, see Alavi, S.A.; Giunti, C. Which is the quantum decay law of relativistic particles? Europhys. Lett. 2015, 109, 60001.

(C) 2016 by the author; licensee MDPI, Basel, Switzerland. This article is an open access article distributed under the terms and conditions of the Creative Commons Attribution (CC-BY) license (http:// creativecommons.org/licenses/by/4.0/). 